\title{
Visualization as an epistemological learning tool: an introduction
}

\author{
F. D. Rivera $\cdot$ Heinz Steinbring $\cdot$ Abraham Arcavi
}

Accepted: 11 October 2013/Published online: 22 October 2013

(c) FIZ Karlsruhe 2013

There are nonvisual ways of forging an isomorphism of words, images or symbols to what they denote, but images are particularly conducive to the essential, dialectical movement between the creative stages of discovery and the deliberative rational stages in which rules and evaluative criteria are introduced to fix meanings and turn images from interpretations into evidence. (Gooding, 2006, p. 60)

This collection of ten research articles and two commentaries explores the mutually determining relationship between visual representations and epistemological experiences in school mathematics. While the eyes (and hands) play a significant role in visual acting on objects, such performance is also and in fact often mediated by discursive contexts in which they arise in the first place. The superficial appearance is that symbols and images conveniently mold together to form an undifferentiated conceptual representation. However, as conveyed in the twelve papers that comprise this special issue, mediated experiences play a central role in linking the visual construction of the mathematical form and its significant other, the mathematical construction of the visual form. Visualization as an epistemological learning tool in mathematics, thus, begins with this strong assumption in which case the minds of learners are not seen as unaided or unconstrained but committed to look in particular ways that support the emergence of necessary mathematical knowledge. Classroom interactions

F. D. Rivera $(\bowtie)$

San Jose State University, San Jose, CA, USA

e-mail: ferdinand.rivera@sjsu.edu

H. Steinbring

Universität Duisburg-Essen, Essen, Germany

\author{
A. Arcavi \\ The Weizmann Institute of Science, Rehovot, Israel
}

that are rooted in sociocultural practices, for instance, can effectively provide grounding for meaningful and purposeful visualization, encouraging learners to engage in visual thinking and reasoning in well-defined structural contexts. Within/against such sociocultural constraints, multiple interpretations are still possible, and so are creative and interesting discoveries. Even visual processing, which enables learners to interact with the external representations of mathematical objects, appears to be a learning progression phenomenon that culminates in inferring and applying valid relationships as a result of thinkering with the concrete forms. That is to say, visual processing engenders learners to "discover the unexpected, and describe and explain the expected" (Thomas \& Cook, 2005).

In this special issue that tackles the role of visualization in mathematical learning, the authors were invited to address the following concerns below.

1. What is the role of visualization in the development of mathematical knowledge?

2. Are there kinds, qualities, and/or hierarchies of visualization and visual skills?

3. What does progressive visualization mean, and how does it emerge in mathematical activity?

4. How do learners of varying levels of ability (i.e., abled students and students with mathematical difficulties) employ visualization in learning mathematics?

5. What visualization strategies do learners employ that enable them to construct meaningful conceptual content?

Two senior scholars, Raymond Duval and Norma Presmeg, whose early work and contributions in this area of research in mathematics education have moved the field forward in significant ways, graciously accepted the important task of providing critical commentaries on the papers around the following areas below. 
1. How is visualization as an epistemological learning tool described in this set of papers?

2. What converging knowledge about visualization in mathematical thinking and learning do we gain from the papers?

3. What (else) is missing? What should we be concerned about after reading the papers? What other questions are pertinent to ask?

4. More importantly, how can we move the field forward in both aspects of theory and methodology?

We hope that the preceding questions and, especially the papers, provide sufficient motivation to incite further discourse in this area of research. The authors' collective experiences drawn from their empirical work with a variety of learners provide compelling empirical evidence of the power of visualization in supporting sensemaking skills and growth in mathematical knowledge and understanding through patterns or structures that learners infer and produce when "the senses (sight, hearing and proprioception), the hands, imagination, systematic and critical reasoning and ... institutions" (p. 43) come together to "show more than the eye could ever see" (Gooding, 2006, p. 54).

\section{References}

Gooding, D. (2006). From phenomenology to field theory: Faraday's visual reasoning. Perspectives on Science, 14(1), 40-65.

Thomas, J., \& Cook, K. (2005). Illuminating the path: The research and development agenda for visual analytics. Washington, DC: National Visualization and Analytics Center. 